\title{
Retrospective semiometrics of the sign valeur
}

\section{[La semiometrie retrospective du signe valeur]}

\author{
Larisa G. Vikulova - Elena G. Tareva - Evgenia F. Serebrennikova - \\ Svetlana A. Gerasimova - Valeriya A. Rayskina
}

DOI: 10.18355/XL.2020.13.01.13

\begin{abstract}
The modern use of the word valeur as a reference notion or a term highlights its status of a sign referring to an archive of knowledge the conceptual formation thereof is applicable to the history of language. By means of semiometrics, a type of semantic diachronic analysis based on the study of representative texts, the paper presents a retrospective evolution of the sign. The paper examines the original generic semantic structure, significant periods of the sign's conceptual transformation in the history of the French language, and the vectors of generalization and differentiation by the anthropological criterion and, taken together, they uncover a tendency of formation of a categorical status sign.
\end{abstract}

Key words: value, retrospective evolution, semiometrics, a categorical status sign, axiology and linguistics

\section{Résumé}

L'emploi actuel du mot valeur en tant que notion d'appui et un terme dans le discours contemporain démontre son statut du signe qui appartient à l'archive du savoir dont la formation conceptuelle se rapporte à l'histoire de la langue. Moyennant la sémiométrie, genre d'analyse à la fois sémantique et diachronique basée sur les textes représentatifs, l'article traite le problème de l'évolution rétrospective du signe. La structure sémantique initiale générative, les périodes de transformations conceptuelles pertinentes dans l'histoire de la langue française ainsi que les vecteurs de la généralisation et de la différentiation selon le critère anthropologique sont établies afin de tracer la tendance du signe vers le terme catégorisant.

Mots-clés: valeur, évolution rétrospective, sémiométrie, signe catégorisant, axiologie et linguistique

\section{Introduction}

Valeur comme terme de base de la linguistique axiologique

Un grand métissage des valeurs au début du XXIe siècle fait réfléchir à la notion même de la valeur en vue de sa délimitation linguistique, philosophique et ethnoculturelle relevant du domaine de l'axiologie (du grec axis - ce qui est précieux, digne d'être estimé) où elle constitue le terme nucléaire. En effet, il est impossible d'appréhender les phénomènes d'ordre personnel, interpersonnel, social ou politique sans identifier leurs fondements axiologiques. De nos jours, dans un dialogue social le discours qui est toujours axé sur un concept reprend de plus en plus de la valeur morale s'agit-il de la campagne électorale, de la crise identitaire nationale, des risques globaux ou de l'application des doubles standards à l'interprétation des faits et des événements. La quête de points de repères est innée à l'époque de mondialisation et d'informatisation qui est la nôtre.

L'axiologie vise à représenter une théorie des valeurs de toute chose, plus précisément, une étude de quelconque sens dans un concept de valeur (Arnaud, 2012). Dans le cadre de la linguistique, l'axiologie traite pour son objet des éléments

XLinguae, Volume 13 Issue 1, January 2020, ISSN 1337-8384, eISSN 2453-711X 
significatifs d'une langue particulière par opposition à la sémantique, qui étudie le sens en général, indifféremment à une langue donnée (Henriette, 2001: 62). Ainsi il est à dégager dans le discours les propositions axiologiques - les jugements de valeur qui accumulent des vérités axiologiques subjectives mais qui restent objectivement valides puisqu'elles se basent sur le raisonnement logiquement correct (Boudon, 1995: 218).

Le champ d'études axiologique est fondé sur un certain nombre d'hypothèses concernant le caractère subjectif ou objectif de l'attitude «valorisante» du sujet d'énonciation, le mode d'évolution des valeurs progressif ou révolutionnaire, la nature des sources des valeurs dans la société. Le système des valeurs est vu dans ses paramètres relationnelle et absolue, économique et politique, féminine et masculine, post-matérialiste ou religieuse, explicite et implicite, directe ou imagée. Depuis l'apparition de la linguistique pragmatique, l'analyse des unités langagières se fait non seulement du point de vue vériconditionnel (relatif aux conditions de vérité de l'énonciation) mais plutôt du point de vue de leur «appropriativité» communicative et de leur contenu axiologique (Pupier, 1998: 51).

On insiste souvent sur l'idée d'enracinement national des valeurs et leurs attachements à une culture concrète. Traditionnellement on distingue deux strates principales de l'axiosphère, l'un portant sur la sphère personnelle (les valeurs de l'espace privé, marquées par l'individualisme et l'autonomie), l'autre sur la sphère nationale / sociale (les valeurs de l'espace public, qui font lien dans la société) (Boudon, Mingasson, 2006: 31). A notre avis, les deux font un continuum conceptuel qui se révèle dans les jugements personnels. L'idée de base peut être formulée ainsi: l'axiosphère a des paramètres objectif et subjectif à la fois et se constitue grâce à l'établissement de l'attitude d'appréciation / évaluation envers l'objet de la pensée. Le paramètre objectif reflète le caractère conventionnel du système des valeurs créé par le peuple étant donné que son appropriation se fait progressivement au cours de la socialisation de l'individu. Le paramètre subjectif s'appuie sur le fait que toute conceptualisation porte en soi une appréciation / évaluation ce qui fait qu'une attitude axiologique trouve une manifestation dans le discours de l'individu dans le cadre de sa condition humaine et des situations de vie c'est-à-dire celles d'énonciation concrète.

Il est clair pourtant que le terme valeur est employé souvent d'une manière aprioriste. Or l'existence d'un nom commun porteur d'une signification de telle ampleur dans la langue révèle avec évidence une catégorisation plus ou moins stable de tout le domaine notionnel que ce terme marque dans le conscient collectif et individuel (Lebart, Piron, Steiner 2003: 5). L'emploi actuel du mot valeur en tant qu'une notion d'appui et un terme dans le savoir humanitaire multidisciplinaire dans le discours philosophique, logique, psychologique, sociologique, pédagogique, économique (système de valeur, valeurs morales, valeurs nationales culturelles, évolution des valeurs, valorisation, valeur ajoutée et autres) démontre son statut du signe qui appartient à l'archive du savoir (Foucault, 1969: 169-170) dont la formation conceptuelle se rapporte à l'histoire de la langue dans ses liens innés avec la pensée et la culture. La question se pose d'essayer de révéler les moments de sa conception initiale dans la langue, des périodes clés des transformations sémantiques ultérieures et des tendances qui ont finalisé son statut actuel notionnel. Alors nous nous posons l'objectif de retracer brièvement l'évolution du nom «la valeur» dans la langue française ayant recours à la sémiométrie du mot dans la rétrospective historique. Le choix de la langue française pour une telle analyse diachronique s'explique par l'étymologie assez transparente du mot français et par une tradition lexicographique et lexiculturelle très riche en France ce qui contribue bien évidemment à une sémiométrie complète des entités langagières en question.

Le mot valeur est caractérisé par sa haute fréquence d'usage: d'après les données du moteur francophone de recherche Google de l'octobre 2018 on compte plus de 147 
millions d'entrées du lexème en question. Dans la liste du Lexique la valeur obtient la fréquence de 152.32 sur 1 million de mots (Lexique - base de donnees lexicales libre, 2018). Pour l'axiosphère de l'homme c'est un mot-clé aussi bien que pour le discours de raisonnement en général (philosophique, sociologique, littéraire, médiatique, ordinaire). L'indicateur du rôle-clé par rapport au mot valeur est son usage en tant que terme central dans le discours philosophique axiologique la sociologie, la psychologie sociale, l'anthropologie, l'histoire, la linguistique, son importance dans la littérature (Boudon, 1995; Kagan, 1997; Pupier, 1998; Henriette, 2001; Lebart, Piron, Steiner, 2003; Boudon, Mingasson, 2006; Teubert, 2009; Arnaud, 2012; Zheltukhina et al., 2016a, 2016b, 2016c, 2017a), son usage dans le langage ordinaire (les enquêtes, les sondages) et dans d'autres sphères du savoir anthropologique.

A l'étape actuelle, il est important, selon le philosophe russe M.S. Kagan (1997), de construire le modèle systémique de l'axiosphère, valable pour toutes les sciences humaines, dont le contenu textuel se compose, selon leur nature, non de connaissances, mais de valeurs (Kagan, 1997: 40). L'analyse linguistique sémiométrique du mot-clé de l'axiosphère permet d'établir les étapes importantes du processus de la formation de la sphère notionnelle correspondante.

Afin de passer à la présentation de la méthodologie de recherche et des résultats de l'analyse sémiométrique, faisons référence à la définition axiologique contemporaine, très juste à notre avis, pour en faire un point de départ dans l'analyse du terme. En s'inspirant des idées du sociologue Jean Stoetzel des années 40, Pierre Bréchon (2003), Président de l'Association pour la recherche sur les systèmes des valeurs (ARVAL) note que «les valeurs sont des idéaux, des préférences qui prédisposent les individus à agir dans un sens déterminé. Elles appartiennent aux orientations profondes qui structurent les représentations et les actions d'un individu» (Bréchon, 2003: 13).

\section{Principes méthodologiques: la sémiométrie et l'analyse de corpus de textes}

La sémiométrie en tant que l'interprétation du terme dans un contexte représentatif, relevant du moment bien précis dans l'évolution de la culture, faisant «autorité » pour une période concrète mais prise dans le continu des autres interprétations du même type, pourrait servir d'un des moyens pertinents afin d'établir l'essentiel de son spectre sémantique au cours du temps pour permettre ensuite de compredre mieux ses fondements ethnoculturels et sociologiques. Une telle méthode en même temps linguistique et sociologique de mesurer un espace du sens des mots vise à décrire des systèmes de valeurs d'une société ou d'un group social défini en édudiant de mots auxquels il est possible d'attribuer une note / une valeur (Lebart, Piron, Steiner 2003: $3,5)$. Ce type d'analyse linguistique permet de révéler le contenu conceptuel, onthologique et axiologique des phénomènes complexes linguoculturels. En conséquent, il devient possible d'étudier l'état d'une société prise dans un cadre historique et culturel précis à travers les mots-clès (mots de civilisation) représentant ses valeurs globales sociales, culturelles et spirituelles.

L'analyse sémiométrique implique la synthèse des significations d'un lexème dans des textes narratifs et scientifiques dans le but d'établir les étapes de l'évolution conceptuelle et de la transformation axiologique de la notion étudiée. La structure de l'analyse sémiométrique se compose des étapes suivantes: l'examen étymologique, l'étude des définitions lexicographiques, l'analyse comparative de descriptions et la détermination des éléments cruciaux construisant le contenu significatif du terme.

Dans l'analyse sémiométrique retrospective du mot valeur nous partons des idées suivantes. Un nom commun à la sémantique généralisante est envisagé en tant que porteur de la notion / du concept. Les concepts reflètent une vision bien particulière de la réalité qui s'ouvre à la pensée dans l'espace culturelle dans une période déterminée.

XLinguae, Volume 13 Issue 1, January 2020, ISSN 1337-8384, eISSN 2453-711X 
Ils jouent le rôle des catégorisateurs qui permettent de regrouper les objets similaires dans des classes correspondantes. C'est grâce aux concepts que les sujets parlants perçoivent, structurent, classifient et interprètent le monde environnant. Le concept ne vient pas directement de la signification du mot, mais il résulte de l'interaction de la définition lexicographique et de l'usage personnel et collectif de ce mot. Sur ce plan, le mot est envisagé dans l'énonciation comme le nom du concept qui peut être actualisé, selon sa nature, par les moyens langagiers divers. Il est évident que le recours à l'évolution de la sémantique du mot dans les contextes concrets permet de mettre en évidence certaines régularités des processus de la réflexion à son contenu aux étapes différentes de l'expérience dans les sphères personnelle et nationale, la formation du domaine notionnel dans la langue.

Les thèses de base que nous dégageons pour l'analyse sémiométrique sont suivantes.

- L'ensemble de toutes les significations d'un mot concret a une structure hiérarchique où il y a des significations hautement représentatives (nucléaires), moins représentatives, marginales et occasionnelles (virtuelles). Ainsi la sémiométrie permettra de faire une carte-espace qui reflète la sphère mentale de la représentation de ce domaine notionnel avec des reliefs plus ou moins expliqués.

- La sémiométrie a un caractère mobile dans le temps chronologique (XIIe siècle, XIIIe siècle, XIXe siècle etc.) et dans les étapes de la représentation du mot / de la notion dans la société en reflétant l'évolution de l'expérience cognitive de l'homme et de la société en général.

- Les critères de la représentativité dans la sémiométrie sont 1/ la fréquence générale de l'usage fixée par le choix de certains corpus de textes ou les indices (le coefficient) d'Internet; 2 le caractère du mot-clé qui a la capacité de servir du moyen de la mise en relief et de l'interprétation des grands fragments du champ notionnel correspondant à une langue concrète. En utilisant la métaphore de «le site» pour le mot-clé, selon la terminologie informatique, nous pouvons dire que l'ouverture de ce site va ouvrir inévitablement son contenu plus vaste et détaillé.

Le critère de la validité de l'étude sémiométrique exige l'analyse des données textuelles volumineuses et diversifiées. Afin de respecter cette prescription méthodologique nous faisons recours aux instruments de recherche et d'analyse de la linguistique de corpus. Parmi de nombreux points de vue sur le terme «corpus» citons la définition traditionnelle de J. Sinclair (2005): «collection of pieces of language text in electronic form, selected according to external criteria to represent, as far as possible, a language or language variety as a source of data for linguistic research». Dans l'adaptation française le corpus est perçu comme «une collection, réglée par des principes, de données du langage empirique, de textes (ou de fragments de textes), qui sont des échantillons d'un discours donné, dotés en conséquence d'une valeur représentative» (Teubert, 2009).

L'utilisation des corpus (corpora) électroniques textuels permet d'augmenter l'efficacité et le matériel du travail vue les outils automatiques du traitement des données linguistiques et la possibilité de réaliser une recherche différenciée (morphologique, syntaxique, sémantique). Les instruments de l'élaboration des statistiques, des concordances, des contextes et des co-occurrences, l'analyse des données textuelles authentiques et l'approche généralement empirique aux phénomènes langagiers permettent d'affirmer la «rigueur scientifique» d'une telle recherche (da Silva Genest, Masson, 2017: 89). Ainsi l'objectif majeur du travail dans le corpus consiste à «valider la cohérence d'une théorie élaborée sur des bases introspectives» (Condamines, 2005: 37). Dans le cadre de cette recherche nous avons utilisé les corpus électroniques textuels suivants:

1) la Base de français médiéval - le corpus rassemblant les textes français du Moyen Âge (IXe-XVe siècles, éditions originales, manuscrits et versions diplomatiques) qui a tout un ensemble d'instruments de recherche et d'analyse: requêtes, calculs de cooccurrence, étiquetage morphosyntaxique etc. Deux ouvrages littéraires sont analysés 
dans ce corpus: la Chanson de Roland et la Queste del saint Graal (Moignet, 2013).

2) le Dictionnaire Electronique de Chrétien de Troyes - le corpus textuel regroupant 5 œuvres du XIIe-XIIIe siècles de l'auteur médiéval Chrétien de Troyes (Érec et Énide, Cligès, Lancelot ou le Chevalier à la Charrette, Yvain ou le Chevalier au Lion et Perceval ou le Conte du Graal). C'est un projet conjoint du laboratoire ATILF (Dictionnaire electronique de chretien de troyes, 2014), du centre CNRS, de l'Université de Lorraine, de l'Université de Provence et de l'Université d'Ottawa. Parmi les instruments du traitement textuel numérique notons la recherche d'une entrée, d'un étymon et d'une forme grammaticale, la lemmatisation, les cooccurrences.

En outre des corpus diachroniques, la matière d'étude sémiométrique a été fournie par des dictionnaires imprimés et des bases lexicales électroniques universellement reconnus qui ne sont pas temporellement spécifiés: Dictionnaire électronique de l'Académie française, Dictionnaire étymologique, Base de Données Lexicales Libre et Dictionnaire historique de la langue française (Le Robert, 1992). De même, les dictionnaires Lexique de l'ancien français et Dictionnaire de l'ancienne langue française et de tous ses dialectes du IXe au XVe siècle ont été utilisés spécifiquement pour l'étude sémiométrique en ancien français (IX-XIII ss.); Dictionnaire du moyen français (1330-1500) pour la période du moyen français et du français classique (XIV-XVIII ss.). Finalement, pour la période du français moderne nous avons exploité les bases textuelles analytiques:

- l'application Ngram (Google books ngram viewer, 2018) viewer sur la plateforme Google books permettant d'étudier l'évolution de la fréquence des lexèmes ou des groupements de mots dans les sources imprimées entre les années 1553 et 2008 pour la base francophone;

- Le grand dictionnaire terminologique (2012) (GDT) dirigé par L'Office québécois de la langue française, qui représente une banque de lexique spécialisé contenant les précisions détaillées concernant le domaine d'emploi du terme en question, la définition, les formes grammaticales et les équivalents dans d'autres langues;

- La fonction du dictionnaire analogique sur le portail encyclopédique en ligne Sensagent de la plateforme LeParisien qui permet d'analyser les champs lexicaux et les taxonomies.

La sémiométrie du signe linguistique a pour le point de départ la notion étymologique dont l'analyse dans la diachronie permet de faire voir l'espace notionnel a partir de l'étape d'origine jusqu'au discours moderne. L'élargissement de l'espace sémantique est déterminé par les mutations sémantiques et les modifications que le mot subit lors de son évolution diachronique. A son tour, la modification de la sémantique est déterminée par les processus importants aux niveaux social et communicatif. Ceci dit, la sémiométrie fait partie de la lexiculture de la langue. Le linguiste a la possibilité d'analyser les changements sémantiques d'après le corpus des contextes représentatifs d'entrées. La sémiométrie du mot valeur est fondée sur les données des dictionnaires variés: étymologique, raisonné, lexiculturel, encyclopédique ainsi que sur les textes des auteurs contemporains et les analytiques des données des corpus textuels d'Internet.

\section{Les résultats de l'analyse sémiométrique de significations du terme valeur en} diachronie

1. La signification primaire, étymologique immédiate déterminant la forme intérieure du mot valeur

L'étymologie du mot valeur a une interprétation double d'après les données des dictionnaires historiques français. Certaines sources témoignent que ce mot remonte à une forme verbale, tandis que d'autres affirment que sa forme primaire provient

XLinguae, Volume 13 Issue 1, January 2020, ISSN 1337-8384, eISSN 2453-711X 
directement du nom. Selon la plupart des dictionnaires, le mot dérive du verbe latin valêre, celui-ci provenant du radical proto-indo-européen wal - être fort (Harper, 2000; Littré, 2009). A noter que le dictionnaire de la langue latine indique la polysémie de ce verbe qui comprend les significations suivantes: devenir fort, vigoureux; être en parfaite santé; être efficace, puissant, agir avec force (Gaffiot, 2016: 1393). En revanche, les dictionnaires de la langue française accentuent différemment une seule signification que, selon eux, a le verbe de départ pour l'ancien français. Par exemple, le dictionnaire de E. Littré (2009) fait remonter la signification primaire de valeur littéralement au verbe latin être fort, bien portant (Littré, 2009): force, courage à la guerre, dans le combat (le sens propre du verbe latin valēre étant être en bonne santé, fort). Le Dictionnaire étymologique marque le même sens primaire en notant que dans le bas latin il existe déjà le substantif valor, valoris.

Une autre étymologie, nominale et non pas verbale, est présentée dans un des nouveaux dictionnaires historiques (DH 1992: 3993): "Valeur» est issu (1080, aussi valur) de «valorem», accusatif du latin classique «valor, valoris» qui a donné l'italien «valore», espagnol "valor» et est dérivé de «valere» (> valoir).

Autrement dit, d'après les auteurs du Dictionnaire historique, le substantif de l'ancien français valeur provient de la forme accusative du substantif qui existait déjà en latin classique. A son tour ce substantif remonte au verbe.

Alors, il paraît important du point de vue conceptuel qu'à la base du sémiosis du signifiant se trouve la forme verbale dont la nature prédicative indique la primauté de l'attribution de la qualité à un objet de la pensée. La compréhension de la nature de la valeur présuppose qu'à la différence des représentations qui marquent le rapport de nominalisation des objets celle de valeur porte initialement en soi l'idée d'établissement de la relation entre le sujet et l'objet.

Les dictionnaires ont fixé l'un des premiers emplois du mot valeur dans La Chanson de Rolland (BFM-CR):

1. Sa grant valor, kil purreit acunter? (1. 534);

2. Que ja pur mei perdet sa valur France! (1. 1090);

3. En tel bataille n'ai cure de bastun; Fers e acers $i$ deit aveir valor (1. 1362);

4. Itel valor deit aveir chevaler Ki armes portet e en bon cheval set (1. 1877).

Dans ce poème épique datant de la fin du XIe siècle (vers l'an 1080) le mot en question à deux variantes orthographiques (valor / valur). Dans les contextes cités valeur signifie: les vertus, les mérite, les qualités d'un chevalier (1, 4); le prix, l'honneur du pays de France (2); l'importance des armes, des seuls objets qui vaillent à la guerre (3).

C'est dans le Dictionnaire étymologique que nous trouvons la réponse aux questions: en quel moment, sous quelle forme et à la base de quel sème a eu lieu le changement de la signification du mot latin. Il s'agit plus précisément de l'évolution de la notion de valere $=$ être bien portant, pas malade observés à côté des autres expressions différentes de celle de départ, par exemple être bien portant, se sentir bien. Toujours le Dictionnaire étymologique fixe le pas suivant dans l'usage du verbe latin: l'apparition du dérivé être évalué = être apprécié. On peut supposer que l'apparition de cette expression a été motivée par les facteurs dont:

1) l'idée de la qualité personnelle positive: la santé, la force, la capacité de l'autoprésentation solide;

2) la possibilité de la gradation dans la manifestation de cette qualité chez l'hommeporteur et, comme suite,

3) la possibilité de s'assurer de la présence de cette qualité et d'exprimer son attitude envers cette qualité.

Autrement dit, dans la sémiométrie du mot en question l'étape suivante est liée au développement à son base de la notion de la portée en corrélation avec la notion de la force et celle de la robustesse.

Il y a lieu d'affirmer déjà au début de l'évolution de l'ancien français que le substantif 
valor, valoris porte la signification modifiée par rapport à l'étymon latin. Il est probable que ce mot a pénétré en anglais (XIe siècle) étant déjà modifié.

Ainsi, la formule suivante peut résumer le changement dans la sémiométrie diachronique du mot analysé à l'étape du passage du latin tardif à l'ancien français:

valere $\rightarrow$ être bien portant $\rightarrow$ être évalué ( $>$ valor, valoris).

\section{La sémiométrie du mot valeur en ancien français (IX-XIII ss.)}

Les témoignages de l'usage de ce mot qu'on trouve dans les textes de la période indiquée montrent la polysémie du substantif et du verbe provenant du verbe latin valere. Ainsi il est possible d'établir une certaine hiérarchie dans leur sémantique.

D'après les données des deux dictionnaires de l'ancien français de F. Godefroy (1901: 526; 1902: 282-283) aussi bien que du dictionnaire de l' ancien français de A.J. Greimas (1969: 653-655), dans la période mentionnée la forme verbale valoir (valer, valair) a la grille suivante des significations:

1) être estimé comme ayant certaine qualité, certain mérite;

2) donner valeur, être estimé à l'égal de quelqu'un, de quelque chose;

3) être estimé comme méritant quelque chose;

4) avoir de la force, être bien portant, fort;

5) servir à, être utile à;

6) défendre par sa valeur;

7) être préférable.

Les termes nominaux au radical du mot valeur (valor, val, valance, value, valiment, valissance, valitude, valoir) est employé avec les significations suivantes:

1) la valeur, le prix, ce que vaux une personne, une chose, ce qu'elle est estimée pour son mérite, ses qualités;

2) la vaillance, la vigueur, l'acte valeureuse, l'action de vaillance;

3) les mérites, les qualités, les perfections;

4) la valeur, la vertu guerrière;

5) la santé, l'état de celui qui se porte bien.

A cette grille plutôt «anthropologique» de significations il est à ajouter d'autres qui témoignent du développement, en cette période, de l'usage «non-anthropologique» voire parfois financier, concernant la référence non aux personnes mais aux choses:

1) l'estimation à un certain prix pécuniaire, à une certaine quantité;

2) le fait de donner force à, valider, légaliser;

3) l'avantage, le profit, la récompense, la fortune;

4) le revenu, la valeur, l'équivalent, ce que vaut une chose pécuniairement;

5) l'expression aveir valur (DH 1992: 3993).

Cet emploi est présent de manière occasionnelle déjà au début du XIIe siècle, par exemple dans le roman anonyme en prose des années 1215-1220 Queste del Saint Graal (Marchello-Nizia \& Lavrentiev, 2013):

1) cest fust ne porroit riens valoir, et ces flors sont assez plus merveilleuses que je ne cuidoie (p. 201a);

2) cil en qui servise tu t'es mis ne puisse plus valoir que tes armes (p. 220b).

Ainsi, dans la sémiométrie générale du mot on peut dégager deux groupes de significations. Ce dernier usage souligné dans le Dictionnaire historique montre que ce mot manifeste le double sémantisme dès le début de son usage jusqu'à nos jours. D'une part, le mot désigne celui qui est digne du respect vu ses mérites: une personne qui a de la valeur. Cette signification est appliquée à la qualité d'une personne. De l'autre part, le mot est utilisé pour désigner la qualité d'un objet: avoir valeur = être propre à un certain usage. La signification référentielle et anthropologique du mot s'élargit de la qualité de la personne à l'importance de la personne.

En ancien français, la signification la plus représentative est celle qui témoigne de la

XLinguae, Volume 13 Issue 1, January 2020, ISSN 1337-8384, eISSN 2453-711X 
vaillance, bravoure (Dubois, Mittérand, Dauzat, 2001). Cette signification prédomine au début et dans la première moitié de l'ancien français. Afin d'exemplifier cette signification, citons certains passages des romans de chevalerie de Chrétien de Troyes (1170-1190), affichés dans le Dictionnaire électronique de Chrétien de Troyes (DECT):

1. Ne que au soloil fet la lune, Ne de biauté ne de valor Ne de franchise ne d'enor (Erec et Enide, 1. 835);

2. Car la valor et la bonté De l'arrabi veü avoient (Cligès, 1. 3574);

3. Ne je ne pans mie ne croi C'onques Dex feïst chevalier Qui se poïst apareillier A ta valor ne a ton pris (Lancelot ou le Chevalier de la Charrette, 1. 3699).

Ce même corpus donne au mot valeur la signification de qualité, en la complétant d'une liste des synonymes: la bonté, la vaillance, la chevalerie, la monte, le pris, la pröece, la noblesse.

Le Dictionnaire Littré montre l'usage du mot dans la chronologie suivante (Littré, 2009):

1. XIe s. Sa gran valor, ki purreit acunter ? (Chanson de Rolland, 1. 534);

2. XIIe s. Mais à dame de valor Doit on penser nuit et jor (Roman du Castelain de Couci et de la dame de Fayel, ch. I); Dame, valour, beauté et courtoisie (ib., ch. $\mathrm{XX)}$;

3. XIIIe s. Il estoit home de grant nom et de grant valeur (Joinville, 1. 218).

Il est à noter que la signification dominante de valeur au sens de bravoure, vaillance sort peu à peu de l'usage du XIIe au XVIe ss., et au XVIIe siècle ce sens est marqué comme vieilli (DH 1992: 3993).

A partir du XIIIe siècle, le mot développe d'une façon intense sa signification nonanthropologique et il est utilisé (à partir de 1260) dans les énoncés liés à la désignation du caractère «mesurable» de l'objet dans l'aspect de sa possibilité et de sa capacité d'être objet de l'échange. Dans cette signification ce mot correspond à l'expression l'équivalent de: valeur d'un bijou, valeur marchande (1549, Estienne, Cit. d'après: DH 1992: 3993).

$\mathrm{Au}$ XIIIe siècle on trouve le mot valoreux qui est interprété par les auteurs du Dictionnaire étymologique comme ce qui a du prix (Dubois, Mittérand, Dauzat, 2001: 799). A noter que la formation de l'adjectif provenant du nom valeur montre que le substantif acquiert un potentiel notionnel suffisant permettant «enlever» son contenu substantiel en guise de signe qui peut être appliqué à d'autres phénomènes. De cette façon, on peut voir la progression de la signification de celle qui est référentiellement fixée in esse à la personne-porteuse de la qualité (la vertu, la vaillance) à celle qui n'est pas référentiellement déterminée in fieri. L'attribution d'un indice s'avère désormais indifférente par rapport à un objet ou à un événement par rapport auquel une attitude valorisante est établie.

Ainsi, on peut déduire que la modification la plus considérable de la sémiométrie du mot en ancien français se caractérise par l'évolution syncrétique de deux significations déterminant:

1) la qualité de l'homme (la force, la vaillance, la bravoure, la vertu) et ensuite de la «portée» de l'homme provenant de ses mérites;

2) la qualité de la chose, son utilité pour un usage et un échange.

\section{La sémiométrie du mot valeur en moyen français et en français classique (XIV- XVIII ss.)}

Le Dictionnaire du Moyen Français (Dictionnaire du moyen français, 1330-1500) donne plusieurs significations du mot valeur pour la période du XIVe au XVIe ss.:

1) ce que vaut une personne ou une chose au regard de ce qui est attendu;

2) ce que vaut une chose pécuniairement;

3) la validité, par exemple, d'un document;

4) la qualité d'une personne ou d'une chose qui la rend digne d'estime, d'intérêt; 
par extension: la qualité morale, le mérite;

5) la valeur guerrière, l'acte de vaillance;

6) la condition sociale élevée (dans les expressions de petite valeur, de grande valeur).

Notons l'apparition d'un nouveau sème de connotation sociale, lié à la noblesse du comportement qui fait référence à la condition élevée dans la société. Ce nouvel aspect de signification apparait à côté des significations préexistantes dans les contextes les plus représentatifs de l'usage du mot en question fournis par Dictionnaire Le Littré:

XIVe s. Forte fut la bataille, nulz homs ne vit greigneur Henriot nomble gent et de noble valeur (Chronique de Bertrand du Gouesclin, v. 16205);

XVe s. Il (Louis XI) congnoissoit toutes gens d'auctorité et de valeur qui estoit en Angleterre, en Espagne (Les Mémoires de messire Philippe de Commynes, ch. I, 1. $10)$;

XVIe s. J'ay toute ma vie beaucoup estimé de vous, mes amis; mais, leur disoit elle, voz valeurs me tiennent aujourd'hui tant obligée à vous aimer... (Beaugué, Guerre d'Ecosse, ch. II, 1. 2).

$\mathrm{Au}$ milieu du XVe siècle apparaît l'antonyme de ce mot: la non-valeur (Dubois, Mittérand, Dauzat, 2001: 799):

1) manque de produit dans une terre, dans une ferme, dans une maison (terre en non-valeur) (Académie, 1932; Littré, 2009);

2) dans une armée, des hommes qu'on ne peut compter ni pour le service de campagne ni pour le combat; les musiciens et les soldats faisant près des officiers le service d'ordonnances (Académie, 1932; Littré, 2009);

3) les incapables dans les professions différentes en général (cet employé est une non-valeur) (ibid);

4) dans le commerce, marchandises qui ne se vendent pas (Académie, 1932; Littré, 2009);

5) dans les finances, certaines créances qu'on n'a pu recouvrer (ibid);

6) état d'une propriété qui ne produit aucun revenu (Dictionnaire du moyen français, 1330-1500)

Ce développement du paradigme de la formation des mots témoigne avec toute évidence de l'existence du domaine notionnel stable lié au mot valeur.

On voit que pendant les périodes qui suivent l'ancien français le mot analysé reste très employé. Vers ce temps-là s'organise un espace sémantique homogène qui forme toute une famille lexicale. Ce domaine sémantique réunit les significations suivantes:

1) avoir une portée;

2) être vaillant, courageux dans le combat; à côté de cette signification apparaît la notion de servir, aider;

3) avoir les mérites, être digne d'estime, ce en quoi une personne est digne d'estime quant aux qualités que l'on souhaite à l'homme dans le domaine moral, intellectuel et professionnel. Cette signification a un caractère généralisant par rapport à celles premières et se distingue par une haute fréquence d'emploi dans les textes littéraires.

Autrement dit, la qualité d'être fort, vaillant dans le combat marque déjà une attitude valorisante. Manifestée dans le combat à la défense de la patrie, cette qualité met à part l'homme porteur de cette qualité, le rend important sur le plan social. A noter que parmi tous les exemples donnés par le Dictionnaire de Littré il y a un seul qui se rapporte à la femme: Mais à dame de valor / Doit on penser nuit et jor (Roman du Castelain de Couci et de la dame de Fayel, ch. I).

Cet exemple permet de dire que le champ référentiel de la notion s'élargit de celle de la vaillance à celle du mérite qui peut se manifester non seulement au champ de bataille mais hors du combat et même dans le compartiment d'une dame. Ainsi le

XLinguae, Volume 13 Issue 1, January 2020, ISSN 1337-8384, eISSN 2453-711X 
lexème en vue de l'analyse accumule dans son sémantisme « anthropologique » non plus seulement le sens guerrier, mais aussi la signification morale ou psychologique liée au caractère et au comportement d'une personne.

Il est important de dire que le mot valeur est employé associativement dans le contexte avec les mots liés à l'appréciation des qualités humaines personnelles: perfection, respect, amour, beauté et d'autres. On peut conclure donc que le mot valeur se rapportant initialement à la sphère personnelle de l'homme avant tout, à ces qualités de combattant s'emploie par extension pour apprécier les qualités humaines en générale et plus spécialement celle prouvées au service "de bonnes actions ", approuvées par la société. Cette évolution sémantique manifeste le procédé de laïcisation du concept de valeur dans la période dite « courtoise » ou celle de bonnes manières de l'histoire sociale française.

$\mathrm{Au}$ XVIIe siècle, on voit l'emploi déjà usuel du mot dans le sens de vaillance, vertu (Littré, 2009):

1. Je suis jeune, il est vrai, mais aux âmes bien nées La valeur n'attend pas le nombre des années (Corneille, Cid, ch. II, 1. 2).

2. La parfaite valeur est de faire sans témoins ce qu'on serait capable de faire devant tout le monde (La Rochefoucauld, Maximes, ch. 216).

La représentation de la signification du mot valeur dans les exemples donnés est envisagée du côté de son contenu primaire et comme dans la citation de La Rochefoucauld on voit la variation de la notion. En même temps, le mot valeur peut prendre le sens d'une force aveugle voire violente si elle n'est pas limitée par la prudence et la précaution:

La valeur n'est qu'une force aveugle et impétueuse, qui se trouble et se précipite, si elle n'est éclairée et conduite par la probité et par la prudence (Fléchier, Turenne).

A partir du XVIIe siècle on voit refléter dans ce mot l'appréciation spécialisée de mesure, le caractère mesurable en tant que susceptible d'être échangé qui traduit le fait sociolinguistique de l'objectivation dans la langue de l'idée de «l'échange», de la mesure liée au développement des processus commercial et industriel dans la société (DH 1992: 3993). Ainsi a lieu l'intensification dans la sémiométrie du mot grâce à l'application des notions similaires pour exprimer les idées de l'appréciation - de la mesure - de l'échange - de la dimension.

C'est à juste titre que les auteurs du Dictionnaire historique notent que cette signification complexe englobant tout le spectre des notions donne à ce mot le potentiel significatif qui a trouvé son expression brillante dans les travaux de F. De Saussure. C'est ainsi que le mot valeur a acquis le statut du terme linguistique.

\section{La sémiométrie du mot valeur en français moderne}

$\mathrm{Au}$ XIXe siècle la famille du mot valeur s'élargit grâce à deux notions: la contrevaleur - terme commercial ayant la signification suivante: valeur donnée en échange de celle que l'on reçoit; et l'antivaleur (l'anti-valeur) - terme de l'éthique signifiant ce qui s'oppose aux valeurs communément reçues (Dubois, Mittérand \& Dauzat, 2001, 799; Littré, 2009; Google books ngram viewer, 2018). Prêtons attention aux paradigmes qui s'instaurent:

la valeur $\rightarrow$ la contre-valeur

la valeur $\rightarrow$ l'antivaleur

La différence dans la sémantique des préfixes négatifs qui forment les mots composés nous permet de dire que ce n'est pas l'absence de la valeur qui devient important pour les processus de la conceptualisation de la notion mais sa manifestation contraire: la contre-valeur existe comme un nom contrairement à «quelque chose sans valeur» fonctionnant comme un tour périphrastique. À son tour, la formation du concept de l'antivaleur présuppose le raisonnement profond sur le contenu, la forme et les limites des valeurs morales aussi bien que l'apparition de la possibilité d'un système éthique contraire à la norme. Au niveau de la représentation il est important de focaliser plutôt 
la manifestation d'un événement contraire, son absence est considérée comme neutre, non évidente dans la plupart des situations, pour la majorité des gens. C'est justement sur le fond neutre que devient possible la mise en relief d'un individu ou d'un objet d'après leur destination ou l'acquisition de la qualité d'une valeur.

Pendant toute la période des XIXe-XXIe siècles, selon le Dictionnaire étymologique (Dubois, Mittérand, Dauzat, 2001: 799), on voit l'apparition de toute une famille de dérivés du mot valeur motivés par le sème de l'appréciation. Ils désignent soit le processus et le résultat de l'attribution de la valeur à un objet / un événement soit le processus et le résultat contraires de leur dévalorisation. D'après l'analyse automatique des données textuelles sur Ngram nous élaborons les statistiques suivantes de fréquences (l'emploi calculé sur un million de mots d'après les points de culmination pour la période des années 1900-2008):

- valoriser / valorisation / valorisant - 0,0026\% (années 2000, 2006);

- revaloriser / revalorisation / revalorisant - 0,0006\% (années 1974, 1986);

- dévaloriser / dévalorisation / dévalorisant - 0,0004\% (années 1976, 1996, 2004).

Le Dictionnaire électronique de l'Académie française témoigne également de l'usage des dérivés suivants: l'adverbe valeureusement comme qualification d'une action faite avec valeur, avec courage; l'adjectif valeureux exprimant les traits de caractère synonymique: brave, vaillant, ayant beaucoup de courage (Académie, 1932).

Selon le Dictionnaire historique, du XVIIIe au XIXe siècles l'évolution de la sémantique se caractérise par le renforcement de l'idée du prix (DH 1992: 3993). Cette tendance s'est manifestée en emploi «absolu» du mot dans l'expression valeur élevée et dans d'autres structures figées:

- Objets de valeur $=$ qui ont du prix

- En valeur = en état d'une chose qui a de la valeur 'prix'

- Etre en valeur pour se vendre aisément et avantageusement et être en état de rapporter

- Mettre en valeur (une terre, une région) = mettre en exploitation, faire produire.

$\mathrm{Au}$ XVIIIe siècle, à la suite de l'évolution de cette tendance, on continue à employer le mot valeur dans sa signification spécialisée non anthropologique comme le nom nombrable: une valeur - des valeurs (DH 1992: 3993). De cette façon, le mot varie son statut linguistique du substantif initialement abstrait au nom concret et nombrable désignant un moyen de l'échange.

Passant aux domaines sémantiques de l'emploi du mot, nous nous servons du Dictionnaire analogique qui regroupe les mots en fonction des relations sémantiques lexicales (Sensagent dictionnaire analogique, 2017), et du Grand dictionnaire terminologique qui fournit les définitions thématiquement réparties (GDT). Ainsi, à partir de sème mesure, dimension le mot valeur est utilisé dans les beaux-arts. Dans la musique (à partir de 1740, selon le Dictionnaire historique) ce mot s'emploie pour la désignation d'une durée relative de la note, de l'intervalle de temps. Parmi les expressions fréquentes citons valeur d'une note de musique, valeur tonale, valeur rythmique. Puis, à partir de 1792, ce mot est employé dans la peinture pour désigner le degré d'intensité du ton claire ou sombre propre à chacune des couleurs de la palette, l'effet d'un ton de couleur: donner de la valeur à un ton, donner plus de valeur à un élément.

Pendant les périodes suivantes, on voit la fixation de ce mot dans son usage terminologique dans le discours économique: «La valeur désigne en économie la qualité d'une chose fondée sur son utilité objective ou subjective, d'où valeur d'usage opposée à valeur d'échange: on parle en finances de valeur ajoutée, d'où taxe à la valeur ajoutée, appelée T.V.A.» (DH 1992: 3993). Dans la même optique la valeur est perçue comme terme de banque et de commerce dans les expressions suivantes: la valeur nominale, la valeur réelle, être en valeur (Académie, 1932). Pendant cette

XLinguae, Volume 13 Issue 1, January 2020, ISSN 1337-8384, eISSN 2453-711X 
période entre en usage l'expression figée d'origine latine ad valorem - selon la valeur marchande. La valeur se dit d'un rapport qui s'établit par l'échange entre deux ou divers produits ou services (Littré, 2009). L'aspect usuel du phénomène en question est souligné dans les expressions déposer des valeurs, créer des valeurs, mettre des valeurs en circulation, où la valeur signifie par métonymie des objets de valeur.

Dans les domaines des finances et de gestion, la valeur devient une qualification représentant l'importance comptable et administrative des documents, des billets de banque etc., par exemple, avec le mot composé papiers-valeurs ayant la signification de titres fiduciaires (GDT). Parmi les expressions antonymiques citons le qualificatif de nulle valeur (Littré, 2009).

En tant que terme des mathématiques, la valeur signifie une quantité exprimée en chiffres et provenant de la résolution d'une équation: la valeur algébrique, valeur approchée, valeur numérique (Académie, 1932). Le Dictionnaire historique marque que le mot entre en usage scientifique «par abstraction des acceptions anciennes, de l'expression numérique ou algébrique d'une qualité» (DH 1992: 3993). En termes non seulement des mathématiques, mais plutôt dans des recherches statistiques, informatiques et sociologiques (qui s'appuient sur les données quantitatives) deux contextes ont la même structure: la valeur d'une donnée / la valeur d'un individu; le deuxième cas témoigne de l'anthropologisation du terme. En outre, la locution la valeur de la vie est devenue une périphrase communément employée pour désigner les êtres vivants dans le cadre d'une étude monétaire afin de définir une somme d'argent qu'une société est prête à payer pour maintenir le niveau moyen de vie d'un individu - la valeur humaine (Sensagent dictionnaire analogique, 2017).

La notion en question garde sa sémantique philosophique et éthique au cours des XIXe-XXIe siècles étant le mot-clé de l'axiologie. Ainsi, la valeur signifie le prix intellectuel, esthétique et moral attaché à un jugement, à un phénomène ou à un objet: être de valeur, attacher de la valeur. Témoignons de l'apparition de l'expression une échelle de valeur échelle des valeurs et système des valeurs désignant l'ensemble de normes, de codes de conduite instaurés dans une société (Sensagent dictionnaire analogique, 2017).

Finalement, la valeur connait un véritable épanouissement avec la formation de la théorie linguistique de Ferdinand de Saussure en 1916 dans le Cours de linguistique générale. La valeur en perçue en linguistique structurale de son point de vue sémantique, ayant l'idée du rapport entre les mots et de leur relation au système de la langue. En d'autres termes, il s'agit de la valeur sémantique, par exemple, la valeur d'un mot est une notion aujourd'hui synonymique à celle d'un sème commun pour deux ou plusieurs mots.

Au cours du XIXe siècle la sphère de l'usage du mot s'élargit grâce à sa sémantique non-anthropologique. Il est utilisé d'une manière intense dans les discours littéraire et courant, le mot acquiert le statut clé dans les discours économique et financier, dans le domaine des beaux-arts en tant que terme de la musique et de la peinture.

L'extension du mot, l'enrichissement de sa sémantique aboutissent à la nouvelle étape de sa sémiométrie: le mot est apte à exprimer des significations abstraites et concrètes à la fois. Employé dans le contexte abstrait, le mot désigne dans les énonciations ce que le jugement personnel trouve vrai, bon selon l'opinion commune (valeurs morales, valeurs littéraires etc.). Ce concept devient clé pour la sociologie et, en général, pour les sciences qui étudient l'homme et la société en liant la notion de la valeur avec le jugement appréciatif et la norme.

\section{Conclusion}

L'aperçu bref de la sémiométrie rétrospective du mot la valeur nous emmène à faire les conclusions suivantes. L'évolution du mot "la valeur» dans la langue française est étroitement liée à l'idée de l'attribution à la personne d'une qualité de la santé, de la force et de la vaillance dans le combat ce qui permet de relever le noyau 
ethnoculturel du concept et comparer le terme français à ses équivalents dans d'autres langues. L'évolution sémantique du signe est ancrée dans les vecteurs de généralisation et de différenciation selon le critère anthropologique.

En développant les dimensions référentielles anthropologique et non-anthropologique du mot le sens prévalant est celui d'intensité variable de la qualité de la personne ou de la chose dans les processus de son identification en gardant toutefois le vecteur du regard valorisant sur le meilleur dans la personne ou la chose. Le contenu de l'attitude d'appréciation / d'évaluation des traits identifiants les qualités de la personne ou des choses au cours du temps varie selon les époques mais en même temps porte un caractère progressif intégral en généralisant de plus en plus l'idée de la qualité attribuée à l'objet d'évaluation. C'est justement ce critère qui permet de délimiter la notion de valeur par rapport aux termes contigus d'évaluation et d'appréciation comme un terme catégorisant ce domaine conceptuel.

L'enrichissement de la sémantique du mot "la valeur» se manifeste le long du développement du français à partir du IXe siècle jusqu'à son emploi dans le discours d'aujourd'hui. D'après les données des bases textuelles et des dictionnaires analogiques, les significations actuelles du mot en question sont liées aux phénomènes anthropologiques et non-anthropologiques des domaines très variés: les beaux-arts, l'économie, la gestion, les statistiques, la sociologie, la réflexion philosophique et éthique, l'analyse du discours ce qui permet d'élargir et d'approfondir le champ d'études connexe de l'axiologie et de la linguistique.

La fréquence de l'emploi du terme en vue d'analyse dans toute sorte de textes est en permanente augmentation. Ceci montre l'intérêt et témoigne de l'actualité et de la pertinence de la valeur en tant qu'une catégorie et un phénomène polyvalent.

\section{Bibliographic references}

ACADEMIE. 1932. Dictionnaire electronique de l'academie française. Lorraine: ATILF - CNRS \& Universite de Lorraine. Available online: https://academie.atilf.fr/8/.

ARNAUD, C. 2012. Axiologie 4.0: proposition pour une nouvelle axiologie. Lyon: PUF. Available online: https://www.axiologie.org. ISBN: 978-2-7466-4295-9.

BOUDON, R. - MINGASSON, L. 2006. Entre valeurs de l'espace privé et valeurs communes. Une articulation complexe. In: Informations sociale, Caisse nationale des Allocations familiales, vol. 1, pp. 26-34. ISSN: 0046-9459

BOUDON, R. 1995. L'objectivite des valeurs. In: L'horizon de la culture. Hommage a Fernand Dumont, Les Presses de l'Univ. Laval et l'Inst. quebecois de recherche sur la culture, Quebec, vol. 13, pp. 217-236. ISBN: 2-7637-7428-8.

BRECHON, P. 2003. Introduction, Les valeurs des Francais. Paris: Armand Colin. ISSN 0023-8368. ISBN 9782200929428.

CONDAMINES, A. 2005. Linguistique de corpus et terminologie. In: Langages, vol. 157, pp. 36-47. ISSN: 0458-726X.

DA SILVA GENEST, CH. - MASSON, C. 2017. L'apport de la linguistique de corpus a l'etude des situations cliniques: l'utilisation de ressources écologiques. In: Studii de lingvistică, vol. 7, pp. 89-112. ISSN: 2248-2547.

DICTIONNAIRE DU MOYEN FRANÇAIS. 1330-1500. Lorraine: ATILF - CNRS \& Université de Lorraine. Available online: http://www.atilf.fr/dmf/

DICTIONNAIRE ELECTRONIQUE DE CHRETIEN DE TROYES. 2014. Ottawa: Université d'Ottawa. Available online: http://www.atilf.fr/dect.

DUBOIS, J. - MITTERAND, H. - DAUZAT, A. 2001. Dictionnaire etymologique. Larousse. Paris: VEUF. DE. ISBN-10: 2035320577.

FOUCAULT, M. 1969. L'archeologie du savoir. Paris: Gallimard. ISBN: 978-2-07026999-0.

XLinguae, Volume 13 Issue 1, January 2020, ISSN 1337-8384, eISSN 2453-711X 
GAFFIOT, F. 2016. Dictionnaire latin-francais: nouvelle édition revue et augmentée, sous la dir. de G. Gréco. Paris: Hachette Education. ISBN: 978-2-9554849-0-6.

GODEFROY, F. 1901. Lexique de l'ancien francais. Paris: Librairie universitaire, française et etrangere. ISBN: 489827752.

GODEFROY, F. 1902. Dictionnaire de l'ancienne langue française et de tous ses dialectes du IXe au XVe siecle. Paris: Librairie Emile Bouiilon. ISBN: 34521391.

GOOGLE BOOKS NGRAM VIEWER. 2018. Available online: https://books.google.com/ngrams. Ngram.

GREIMAS, A.J. 1969. Dictionnaire de l'ancien français jusqu'au milieu du XIVe siècle. Paris: Librairie Larousse. ISBN: 460534545.

HARPER, D. 2000. The Online Etymology Dictionary, Lancaster. Available online: https://www.etymonline.com/. OED.

HENRIETTE, W. 2001. Axiologie et semantique chez Andre Martinet. Paris: PUF. ISBN: 9782130520474.

KAGAN, M.S. 1997. Philosophical value theory - La theorie philosophique des valeurs -Philosofskaya théoria tsennosti. St. Peterburg: TOO TK "Petropolis". ISBN: 5-86708-101-X.

LE GRAND DICTIONNAIRE TERMINOLOGIQUE. 2012. Office québécois de la langue française, Montréal. Available online: http://gdt.oqlf.gouv.qc.ca. GDT.

LE ROBERT. 1992. Dictionnaire historique de la langue française. Sous la dir. d'Alain Rey, Dictionnaire Le Rober. Paris: DH. ISBN: 2-85036-187-9.

LEBART, L. - PIRON, M. - STEINER, J.-F. 2003. La semiometrie. Essai de statistique structural. Paris: Dunod. ISBN: 2-10-008105-5.

LEXIQUE - BASE DE DONNEES LEXICALES LIBRE. 2018. Université Savoie MONT BLANC, RISC, CNRS. Available online: http://www.lexique.org/listes.

LITTRÉ, E. 2009. Dictionnaire Le Littré 2.0: logiciel à source ouverte pour consulter hors-ligne, éd. de M. Descerisiers, Free Software Foundation. Available online: http://code.google.com/p/dictionnaire-le-littre/.

MARCHELLO-NIZIA, Ch - LAVRENTIEV, A. Queste del saint graal. 2013., Equipe BFM, Lyon. Available online: http://catalog.bfm-corpus.org/qgraal_cm.

MOIGNET, G. 2013. Chanson De Roland. Paris: Lyon. ISBN: 2040160116

PUPIER, P. 1998. Une première systématique des évaluatifs en français». In: Revue québécoise de linguistique, vol. 26, n. 1, pp. 51-78. ISSN: 0710-0167.

SENSAGENT DICTIONNAIRE ANALOGIQUE. 2017. Available online: ttp://dictionnaire.sensagent.leparisien.fr/index.html.

SINCLAIR, J. 2005. Corpus and Text: Basic Principles. Oxford: Oxbow Books. ISBN: 1-8421-7205-0.

TEUBERT, W. 2009. La linguistique de corpus: une alternative. In: Semen: Revue de sémio-linguistique des textes et discours. Besançon: Presses universitaires de Franche-Comté, pp. 185-200. Available online: http://journals.openedition.org/semen/8923 ISBN: 978-2-84867-256-4.

ZHELTUKHINA, M.R. - BIRYUKOVA, E.V. - GERASIMOVA, S.A. - REPINA, E.A. - KLYOSTER, A.M. - KOMLEVA, L.A. 2017a. Modern Media Advertising: Effective Directions of Influence in Business and Political Communication. In: Man in India, vol. 97, n. 14, pp. 207-215. ISSN: 0025-1569.

ZHELTUKHINA, M.R. - KRASAVSKY, N.A. - SLYSHKIN, G.G. PONOMARENKO, E.B. 2016a. Utilitarian and Aesthetic Values in the Modern German Society (Through the Example of Print Media Advertisements). In: IEJMEMathematics Education, vol. 11, n. 5, pp. 1411-1418. ISSN: 1306-3030

ZHELTUKHINA, M.R. - VIKULOVA, L.G. - SEREBRENNIKOVA, E.F. GERASIMOVA, S.A. - BORBOTKO, L.A. 2016b. Identity as an Element of Human and Language Universes: Axiological Aspect. In: International Journal of Environmental and Science Education, vol.11, n. 17, pp. 10413-10422. eISSN 13063065 . 
ZHELTUKHINA, M.R. - VIKULOVA, L.G. - SLYSHKIN, G.G. - VASILEVA, E.G. 2016c. Naming as Instrument of Strengthening of the Dynastic Power in the early middle Ages (France, England, Vth - XIth Centuries). In: International Journal of Environmental and Science Education, vol. 11, n. 14, pp. 7195-7205. eISSN 13063065.

Words: 8216

Characters: 54286 (30,16 standard pages)

prof. Larisa Georgievna Vikulova, Dr.Sc.

Foreign Languages Institute

Moscow City University

5 b Maly Kazenny per.

105064 Moscow

Russia

vikulovalg@mail.ru

prof. Elena Genrikhovna Tareva, DrSc.

Foreign Languages Institute

Moscow City University

5 b Maly Kazenny per.

105064 Moscow

Russia

elenatareva@mail.ru

prof. Evgenia Fedorovna Serebrennikova, Dr.Sc

Institute of Philology, Media Communications and Foreign Languages

Irkutsk State University

$1 \mathrm{~K}$. Marx str.

664003 Irkutsk

Russia

serebr_ef_76@mail.ru

assoc. prof. Svetlana Anatolyevna Gerasimova, $\mathrm{PhD}$

Foreign Languages Institute

Moscow City University

5 b Maly Kazenny per.

105064 Moscow

Russia

gerasvetlana@yandex.ru

Valeriya Aleksandrovna Rayskina

Foreign Languages Institute

Moscow City University

5 b Maly Kazenny per.

105064 Moscow

Russia

rayskinav@mail.ru 